chemists, biochemists, pharmacologists or physiologists. All these will find some part of the book where they will feel at home, and this should encourage them to see their contribution in relation to that of others. Although it may be difficult to write a book all of which will appeal to readers with such different backgrounds, the subject is not divided up in the way that scientists are. This book is important because it attempts to review synaptic events as a whole.

R. B. Barlow

R. B. Barlow is Lecturer in Chemical Pharmacology at the Medical School, University of Bristol, $U K$.

\section{Recognising variable patterns}

Syntatic Pattern Recognition: Applications. Edited by K. S. Fu. Pp.xi+270. (Springer: Berlin and New York, 1977.) DM 85; $\$ 37.40$.

THIS collection of ten papers by different authors is concerned with the application of automatic pattern recognition to electrocardiograms, speech, chinese ideograms, mathematical expressions, finger prints, remotely sensed imagery and parts of industrial assemblies. The central technical problem is that machines are required to recognise patterns that are not unique. For instance, countless minutely different waveforms should be recognised as clinically normal electrocardiograms. Similarly, two successive finger prints from one right thumb will generally be slightly different, depending for instance on distortion of the skin by pressure while the print is taken. Such differences often make it unexpectedly difficult to make machines recognise what the brain can easily recognise.

There is at least a tenuous analogy between deciding which patterns are bona fide finger prints of one right thumb and deciding which strings of symbols constitute a syntactically admissible sentence in a formal language. In both cases a large or infinite set of strings or patterns has to be defined by means of definitory apparatus that contains relatively little information. Furthermore, a finger print consists for instance of a whorl or tented arch which in turn consists of minutae in various positions; and similarly a sentence consists of phrases which in turn consist of words. This illustrates the general idea that patterns, as well as sentences, can usefully be subject to a hierarchical structural description as in parsing. Because formal language theory is well understood, various workers have tried since about 1964 to do some good with it in automatic pattern recognition.

Chomskian grammars turn out to be too abstract, too simple, for immediate application to pattern recognition. First, a phrase structure is concerned only with concatenation of symbols, not with the details of their relative positions and shapes. Second, a simple grammar is one-dimensional, whereas finger prints are not. Both of these problems can be overcome to some extent, and the book under review gives usually ad hoc details for the different practical applications.

The first chapter is a consise introduction by $\mathrm{Fu}$, whose textbook Syntactic Methods in Pattern Recognition (Academic Press, 1974) makes an even better introduction. The standard of the remaining papers is rather higher than in the usual journals, and several contain useful introductions and bibliographies for their application areas. Stallings' paper on Chinese ideograms is particularly good in this respect.

In pattern recognition one can either start with a technique and see how it works in a given application, or one can start with an application and devise suitable techniques. The present book mainly follows the first of these strategies, whereas the papers in Digital Picture Analysis, Edited by A. Rosenfeld (Springer, 1976) mainly follow the second strategy and turn out not to be straightforwardly syntactic in the pure linguistic sense. The syntactic approach is just one among many; and it has perhaps met its greatest commercial success in reading machines such as the IBM 1287 which are not mentioned in the book under review.

This book is not about established commercial applications but instead reports research explorations of conceivable applications for syntactic techniques, and does not give much idea of the relative efficacy of syntactic compared with other techniques. Nevertheless, it is as good a collection of papers as we are likely to get in 1977 on this topic.

\section{J. R. Ulimann}

J. R. Ullmann is Professor of Computing Science in the Department of Applied Mathematics and Computing Science, University of Sheffield, UK.
BOOKS

ON PURE

AND APPLIED SCIENCE

Books reviewed or men-

tioned in this journal are

available from stock.

Catalogues on application.

Please state interests.

\section{SCIENTIFIC LIBRARY}

ANNUAL SUBSCRIPTION from $\mathbf{f 5 . 0 0}$

Reduced rates for multiple subscriptions Available in U.K. only

Prospectus free on request

\section{H. K. LEWIS \& Co. Ltd. \\ LONDON: 136 GOWER STREET, WC1E 6BS}

Telephone : $01-3874282$

Circle No. 17 on Reader Enquiry Card.

The medium for short reports on all aspects of microbiology and microbial chemistry

FEMS

Microbiology Letters

Chief editor: D.W. Tempest, Amsterdam, The Netherlands

1977 - Volumes 1 and 2 in 12 issues US \$ 81.75/Dfl. 200.00

Published on behalf of the Federation of European Microbiological Societies by

For a specimen copy write to: ELSEVIER/NORTH HOLLAND BOMEDICAL PRESS

P.O. Box 211, Amsterdam,

The Netherlands

The Dutch guilder price is definitive. US $\$$ prices are subject to exchange rate fluctuations. 